\title{
Perfil clínico dos pacientes atendidos na Clínica de Dentística da UFCG
}

Clinical profile of patients at the UFCG Operative Dentistry Clinic

Perfil clínico de los pacientes atendidos en la clínica de operatória dental de UFCG

Maria das Graças Duarte de ANDRADE NETA ${ }^{1}$

José Henrique de Araújo CRUZ $^{1}$

Moan Jéfter Fernandes COSTA $^{1}$

Elizandra Silva da PENHA ${ }^{2}$

Maria Angélica Satyro Gomes ALVES ${ }^{2}$

Abrahão Alves de OLIVEIRA FILHO²

Camila Helena Machado da Costa FIGUEIREDO²

Darlene Cristina Ramos Heloy DANTAS ${ }^{3}$

Gymenna Maria Tenorio GUÊNES ${ }^{2}$

${ }^{1}$ Cirurgião-Dentista pelo Curso de Graduação em Odontologia. Centro de Saúde e Tecnologia Rural, Universidade Federal de Campina Grande, UFCG 58708-110 Patos - PB, Brasil

${ }^{2}$ Professor(a) Doutor(a) do Curso de Graduação em Odontologia. Centro de Saúde e Tecnologia Rural, Universidade Federal de Campina Grande, UFCG 58708-110 Patos - PB, Brasil

${ }^{3}$ Professora Doutora do Curso de Graduação em Odontologia. Departamento de Dentística, Universidade Estadual da Paraíba UEPB 58429-500 Campina Grande - PB, Brasil

\begin{abstract}
Resumo
Para um correto planejamento em saúde e organização em serviço, analisar o perfil e a história clínica dos pacientes atendidos é fundamental, pois esse modelo de estudo se faz necessário para conhecer, através de registros clínicos, as peculiaridades dos pacientes, e também avaliar a qualidade do serviço, compreendendo e atendendo as verdadeiras necessidades da população. Diante desse ponto de vista, o objetivo deste estudo foi traçar o perfil clínico dos pacientes atendidos na clínica de Dentística da Universidade Federal de Campina Grande, do campus Patos PB. Este estudo foi do tipo transversal, observacional, com abordagem indutiva e procedimento comparativo, descritivo, adotando como estratégia de coleta de dados equivalente a 200 prontuários dos pacientes atendidos na clínica escola de Dentística. Considerando os dados, verificou-se que a maior parte dos pacientes eram do gênero feminino (57,5\%), com idades entre 31 e 45 anos (38,5\%), cujo perfil clínico apresentava dentes hígidos (40,75\%), cárie $(19,6 \%)$ e dentes ausentes (18,51\%), ainda, constatou-se que a maior parte dos pacientes não concluíram o tratamento indicado (60\%). Esta pesquisa nos permitiu compreender com mais detalhes os pacientes recebidos nesta clínica escola, demonstrando que um grupo considerável de pacientes possui problemas dentários, e isto é reflexo da realidade quando nos referimos à compreensão das necessidades do paciente no atendimento odontológico, especialmente levando em consideração a falta de acesso da população aos serviços odontológicos de qualidade.
\end{abstract}

Descritores: Odontologia; Dentística Operatória; Perfil de Saúde.

\section{Abstract}

For correct health planning and in-service organization, analyzing the profile and clinical history of the patients seen is essential, as this study model is necessary to know, through clinical records, the peculiarities of the patients, and also to evaluate the quality service, understanding and meeting the real needs of the population. Given this point of view, the objective of this study was to outline the clinical profile of patients seen at the Dentistry clinic of the Federal University of Campina Grande, on the Patos PB campus. This was a cross-sectional, observational study, with an inductive approach and a comparative, descriptive procedure, adopting as a data collection strategy equivalent to 200 medical records of patients seen at the Dentistry school clinic. Considering the data, it was found that most patients were female (57.5\%), aged between 31 and 45 years (38.5\%), whose clinical profile had healthy teeth $(40.75 \%)$, caries $(19.6 \%)$ and missing teeth $(18.51 \%)$, yet, it was found that most patients did not complete the indicated treatment $(60 \%)$. This research allowed us to understand in more detail the patients received at this school clinic, demonstrating that a considerable group of patients have dental problems, and this is a reflection of the reality when it comes to understanding the patient's needs in dental care, especially considering the lack of population access to quality dental services.

Descriptors: Dentistry; Dentistry, Operative; Health Profile.

\section{Resumen}

Para una correcta planificación de la salud y organización en el servicio, es esencial analizar el perfil y la historia clínica de los pacientes atendidos, ya que este modelo de estudio es necesario para conocer, a través de los registros clínicos, las peculiaridades de los pacientes y también para evaluar la calidad. servicio, comprensión y satisfacción de las necesidades reales de la población. Dado este punto de vista, el objetivo de este estudio fue describir el perfil clínico de los pacientes atendidos en la clínica de Odontología de la Universidad Federal de Campina Grande, en el campus de Patos PB. Este fue un estudio transversal, observacional, con un enfoque inductivo y un procedimiento descriptivo comparativo, que se adoptó como una estrategia de recopilación de datos equivalente a 200 registros médicos de pacientes atendidos en la clínica escolar de Odontología. Considerando los datos, se encontró que la mayoría de los pacientes eran mujeres (57.5\%), con edades entre 31 y 45 años (38.5\%), cuyo perfil clínico tenía dientes sanos (40.75\%), caries (19.6\%) y dientes faltantes (18.51\%), sin embargo, se encontró que la mayoría de los pacientes no completaron el tratamiento indicado (60\%). Esta investigación nos permitió comprender con más detalle a los pacientes recibidos en esta clínica escolar, lo que demuestra que un grupo considerable de pacientes tiene problemas dentales, y esto es un reflejo de la realidad cuando se trata de comprender las necesidades del paciente en el cuidado dental, especialmente teniendo en cuenta el falta de acceso de la población a servicios dentales de calidad.

Descriptores: Odontología. Operatória Dental; Perfil de Salud.

INTRODUÇÃO

Conforme Adriola et al. $^{1}$ analisar a história clínica e o perfil dos pacientes atendidos é fundamental para o planejamento em saúde e organização em serviço, onde os dados epidemiológicos nos mostram a viabilidade de quantificar os grupos populacionais e com isso obter os índices de prevalência e incidência das alterações que envolvem a saúde, já que para os estudos de análise dos atendimentos ambulatoriais é preciso a qualidade dos 
registros clínicos, conhecer as peculiaridades dos pacientes e também avaliar a qualidade do serviço, para assim compreender e atender as verdadeiras necessidades da população.

Domingos et al. $^{2}$ afirma que a formação de novos profissionais da Odontologia deve ocorrer de tal maneira que estes assentem o seu trabalho também conforme as necessidades da sociedade e não apenas nos seus entendimentos técnicos ou no interesse de uma resposta financeira, tendo em vista que em épocas passadas os ensinamentos eram tão somente tecnicistas e direcionado para 0 mercado de trabalho, sendo que esse tipo de perfi profissional não atendia as verdadeiras necessidades da população.

Deste modo, é essencial reunir informações sólidas acerca da condição socioeconômica dos pacientes para que se tenha uma exata investigação de suas necessidades, com o intuito de melhorar o atendimento da população ${ }^{1}$.

Para Amorin et al. $^{3}$ independente do reconhecimento da importância da saúde bucal, a assistência odontológica apresentada em serviços públicos no Brasil, não é o bastante para suprir a uma demanda muito grande de pacientes, ocasionando, assim, mínimas possibilidades para a prática da prevenção, da detecção e do possível tratamento precoce da cárie dentária, entre outras afecções bucais.

Segundo García-Pérez et al. ${ }^{4}$ a cárie é considerada uma doença multifatorial, que não está só limitada em exclusivamente a alteração da estrutura dental. Mota-Veloso et al. ${ }^{5}$ afirma também que assa doença causa também sintomatologia dolorosa e até modificação na linguagem oral, afetando a qualidade de vida e a percepção estética, pertinente aos sintomas e sinais demonstrados.

Embora tenha acontecido uma diminuição da ocorrência de cáries dentárias, ainda há uma prevalência bem significante em grande parte da população mundial, este dado expande ainda mais quando se refere aos mais carentes, assegura Marcenes et al. ${ }^{6}$ e muitas vezes essas lesões ocasionam uma coloração indesejável e antiestética, podendo ser vista a olho nu, podendo trazer dor de acordo com Priyank et al. ${ }^{7}$.

Além do que, em conformidade com Gonzales et al. ${ }^{8}$ a perda precoce dos primeiros molares permanentes aponta como a promoção e prevenção da doença cárie sendo ineficaz, e que dentro das consequências da perda prematura do primeiro molar permanente se descobrem alterações na função mastigatória, a continuidade da erupção dos dentes antagonistas, dando abertura para sua extrusão e rotação dos molares adjacentes.

Os tratamentos restauradores são procedimentos básicos que possuem espaço na odontologia preventiva e conservadora. Manter os elementos dentários na cavidade bucal de forma funcional e estético é o seu principal objetivo, vista à grande demanda de pacientes que procuram o serviço de Dentística da Clínica Escola de Odontologia da Universidade Federal de Campina Grande (UFCG). O objetivo deste estudo foi traçar o perfil clínico dos pacientes atendidos na clinica de Dentística da referida instituição de ensino e quantificar a perda do primeiro molar permanente para, epidemiologicamente, averiguar o quadro de pacientes sem este elemento dentário que por diversas vezes é perdido tão precocemente e traçar possíveis medidas preventivas e interceptativas.

\section{MATERIAL E MÉTODO}

O presente estudo foi do tipo transversal, observacional, com abordagem indutiva e procedimento comparativo, descritivo, adotando como estratégia de coleta de dados os prontuários da clínica escola de Dentística da UFCG, realizada por apenas um pesquisador. $O$ município em questão se constitui num centro polarizador de uma vasta região interiorana do Estado, em torno do qual gravitam 50 municípios, e para o qual convergem os interesses de uma parcela bastante significativa da população. Para este trabalho, selecionou-se a Universidade Federal de Campina Grande instituição de Ensino Superior pública, Campus Patos-PB.

O universo foi composto por 500 prontuários de Dentística, após as análises, obteve-se uma amostra de 200 prontuários por meio dos critérios de exclusão como: prontuários que não foram preenchidos e possuíam informações insuficientes para análise dos dados, pacientes menores de 18 anos e prontuários que não foram preenchidos entre os anos de 2014 e 2018. A ficha clínica das disciplinas de Dentística tem a finalidade de acompanhar o plano de tratamento e evolução do paciente, nela contém informações de anamnese e queixa principal que o levou a buscar o atendimento odontológico.

As variáveis de coleta partiram do aspecto sóciodemográfico, como gênero, idade e seguiram com a análise das queixas principais, exames dentários como: dentes hígidos, dentes com restauração em resina composta, dentes com restauração de amálgama, fraturas dentárias, lesões cervicais não cariosas, restaurações temporárias, dentes 
ausentes e perda do primeiro molar; avaliou-se ainda a conclusão do tratamento planejado.

Após coletados, os dados foram registrados na forma de banco de dados do programa estatístico SPSS (Statistical Package for Social Sciences) para Windows 7, versão 23 para análise estatística e inferencial. Para tanto foram analisadas e comparadas as porcentagens e utilizado o teste estatístico do Qui-quadrado para testar a significância entre as variáveis de análise de acordo com o $p$-valor $0,05^{\circ}$. O projeto foi submetido e aprovado pelo Comitê de Ética sob o CAAE: 05312918.0.0000.5181.

\section{RESULTADOS}

Os resultados obtidos através da análise de 200 prontuários de pacientes atendidos na Clínica Escola de Odontologia da Universidade Federal de Campina Grande (UFCG) foram apresentados de modo descritivo e em tabelas para melhor compreensão.

No que concerne ao gênero, a Tabela 1 confirma o número feminino sendo mais prevalente, representando $57,5 \%$ (115) do universo, enquanto o gênero masculino $42,5 \%$ (85). No que diz respeito à faixa etária, também na tabela 1, apresenta a divisão de três grupos distintos, entre 18 a 30 anos, entre 31 a 45 anos e entre 46 a 68 anos, a maioria dos pacientes pertenciam ao intervalo de 31 e 45 anos $(38,5 \%)$.

Tabela 1. Características sociodemográficas dos pacientes atendidos na clínica de dentística com relação ao gênero e idade

\begin{tabular}{|c|c|c|}
\hline VARIÁVEL & No & $\%$ \\
\hline \multicolumn{3}{|l|}{ Sexo } \\
\hline Feminino & 115 & $57,5 \%$ \\
\hline Masculino & 85 & $42,5 \%$ \\
\hline \multicolumn{3}{|l|}{ Faixa Etária } \\
\hline $18-30$ & 75 & $37,5 \%$ \\
\hline $31-45$ & 77 & $38,5 \%$ \\
\hline $46-68$ & 48 & $24 \%$ \\
\hline
\end{tabular}

Durante anamnese, questionou-se sobre a queixa principal e foram relatadas 8 tipos de queixas diferentes, entre as 200 fichas avaliadas. A mais citada foi por motivos de "Restauração" com 42\% (84) e a menos citada foi por presença de cálculo com apenas $1 \%$ (02). Além destes, foram citadas: avaliação de rotina com 18,5\% (37), extração com 1,5\% (03), fratura dentária com 14\% (28), dor com 18,5\% (37), presença de diastema com 2,5\% (05), e limpeza 2\% (04), representados na Tabela 2.

A Tabela 3 expõe, separadamente, as variáveis que define o perfil clínico do paciente, de acordo com o gênero feminino, verificou-se uma maior quantidade da presença de Dentes Hígidos com 38,7\% (1455) do que de dentes Restaurados ou acometidos por Cárie, Fraturas
Dentárias ou Lesões Cervicais Não Cariosas. Os números de Restaurações de Amálgama foram maiores com 9,7\% (364) comparados com resina composta que foi $9,1 \%$ (345). Os dentes ausentes foram representados por $20,7 \%$ (778).

Tabela 2. Variáveis sobre o motivo da procura do atendimento odontológico na clínica escola da UFCG

\begin{tabular}{r|c|c}
\multicolumn{1}{l|}{ VARIÁVEL } & No & \% \\
\hline \multicolumn{2}{l|}{ Queixa Principal } \\
\hline Restauração & 84 & $42 \%$ \\
\hline Avaliação de Rotina & 37 & $18,5 \%$ \\
\hline Dor & 37 & $18,5 \%$ \\
\hline Fratura Dentária & 28 & $14 \%$ \\
\hline Diastema & 5 & $2,5 \%$ \\
\hline Limpeza & 4 & $\mathbf{2 \%}$ \\
\hline Extração & 3 & $1,5 \%$ \\
\hline Presença de cálculo & 2 & $1 \%$ \\
\hline
\end{tabular}

Com relação ao gênero masculino, apresentado na Tabela 3 , os dentes hígidos $43,5 \%$ (1153), dentes cariados 19,4\% (730) e dentes ausentes 15,3\% (407) também prevaleceram. Porém, o que mais chamou atenção foi 0 fato de o gênero masculino, mesmo em menor número, apresentou uma maior quantidade de dentes fraturados 1,6\% (44) e presença de lesões cervicais não cariosas 4,2\% (112) em relação ao gênero feminino que procurou mais atendimento.

Tabela 3. Dados em relação ao perfil clínico analisado de forma separada para o sexo feminino e masculino.

\begin{tabular}{l|c|c}
\hline VARIÁVEL & $\begin{array}{c}\text { Masculino } \\
\text { N(\%) }\end{array}$ & $\begin{array}{c}\text { Feminino } \\
\text { N(\%) }\end{array}$ \\
\hline Dentes Hígidos & $1153(43,5)$ & $1455(38,7 \%)$ \\
\hline Dentes ausentes & $524(19,7)$ & $778(20,7 \%)$ \\
\hline Cárie & $407(15,3)$ & $730(19,4 \%)$ \\
\hline $\begin{array}{l}\text { Restaurações de Amálgama } \\
\text { Dentário }\end{array}$ & $205(7,7)$ & $364(9,7 \%)$ \\
\hline Restaurações de Resina & $194(7,3)$ & $345(9,1 \%)$ \\
\hline $\begin{array}{l}\text { Lesões Cervicais Não } \\
\text { Cariosa }\end{array}$ & $112(4,2)$ & $34(0,9 \%)$ \\
\hline Fratura Dentária & $44(1,6)$ & $31(0,8 \%)$ \\
\hline Restaurações temporária & $9(0,3)$ & $15(0,4 \%)$ \\
\hline
\end{tabular}

No que diz respeito à finalização do tratamento na Clínica de Dentística, analisando a ficha de planejamento e em seguida comparando com os procedimentos executados, os prontuários femininos nos responderam que $63,4 \%$ não concluíram o tratamento mediante planejamento. Semelhante foi o resultado nos prontuários masculinos, 64,7\% não concluíram o tratamento. Contudo, a Tabela 4 mostra que não houve diferença significativamente estatística na análise dos sexos com a conclusão do tratamento ( $p$-valor 0,858 ).

Tabela 4. Associação entre os sexos e a conclusão do tratamento planejado.

\begin{tabular}{r|l|l|l|l}
\hline VARIÁVEL & $\begin{array}{l}\text { Sim } \\
\text { N (\%) }\end{array}$ & $\begin{array}{l}\text { Não } \\
\text { N (\%) }\end{array}$ & $\begin{array}{l}\text { Valor } \\
\mathbf{X}^{\mathbf{2}}\end{array}$ & $\begin{array}{l}\text { p- } \\
\text { valor }\end{array}$ \\
\cline { 1 - 3 } CONCLUSÃO DO TTT & \multirow{3}{*}{0,032} & \multirow{2}{*}{0,858} \\
\hline Feminino & $42(36,52)$ & $73(63,47)$ & \\
\hline Masculino & $30(35,29)$ & $55(64,7)$ & & \\
\hline
\end{tabular}




\section{DISCUSSÃO}

O ensino odontológico para Antunes et $\mathrm{al}^{\cdot 9}$ tem sido característico e tradicional. Durante muito tempo, utiliza-se um modelo biologicista, individualista e mecanicista. $O$ estudante de Odontologia, de acordo com esse modelo, curava em frações, ou melhor, não visualizava o paciente como um todo. A técnica odontológica aplica à visão do usuário do serviço de saúde, e apenas os aspectos mecânicos e curativos eram apontados como essenciais.

Diante disso, vários estudos como este vêm sendo efetuados nas Clínicas Escolas e Serviços de Saúde, para que de fato, os principais motivos da procura do atendimento odontológico sejam principalmente por prevenções de doenças como a cárie e a doença periodontal, pois através desse tipo de pesquisa é reconhecido o perfil clínico de modo geral e também individual de cada paciente, para que assim possam ser estabelecidos os planejamentos e procedimentos adequados para determinadas demandas.

Verificou-se que a maioria da população que procurou pelo atendimento odontológico na clínica-escola da UFCG eram mulheres. A mesma tendência foi observada nos estudos de Kanegane et al. ${ }^{10}$ e Silva et al. ${ }^{11}$ onde avaliaram o perfil dos pacientes atendidos em Pronto Atendimento (PA) com urgências odontológicas. Esta pesquisa constatou que o gênero feminino é o mais prevalente quando se trata em procurar atendimento odontológico, sugerindo que as mulheres possuem uma maior preocupação com a saúde bucal, em detrimento do gênero masculino.

Corroborando com este estudo, Flumingnan et al. ${ }^{12}$, Xavier ${ }^{13}$ e Reis et al. ${ }^{14}$ determinaram que o gênero feminino é predominância na procura do atendimento odontológico. Esta realidade é explicada por as mulheres possuírem uma maior preocupação com a saúde, segundo Ferreira-Junior ${ }^{15^{3}}$ e Gomes $^{16}$ cita que estudos realizados pelo ministério da saúde afirmam que os homens buscam menos por atendimento odontológico nos serviços de saúde, e o principal motivo seria - receio da descoberta de alguma doença considerada grave.

No que diz respeito à faixa etária, o presente estudo demonstrou que os pacientes que mais procuraram atendimento odontológico foram entre 31 e 45 anos de idade. A partir disso, sugere-se que durante essa faixa etária os pacientes possuem 0 hábito de só procurarem o atendimento odontológico de forma curativa quando há dor, enquanto os de idade inferior estão vivendo uma era conhecida como Odontologia Preventiva, que visa evitar possíveis progressos dos problemas que comprometem a saúde bucal.

Em paralelo com o que foi encontrado em nosso estudo, a pesquisa de Domingos et al. ${ }^{2}$ que trata do perfil sóciodemográfico dos pacientes atendidos em uma Clínica Escola de Odontologia, a faixa etária estava entre 41 e 50 anos. Silva e Saintrain ${ }^{17}$ afirmam que pacientes idosos, com idade superior a 60 anos, precisam de um tratamento odontológico mais amplo, já que possuem agravantes na cavidade oral, como perda dental devido à cárie e/ou doença periodontal, sendo uma grande justificativa para exodontia, assim como os problemas endodônticos como necrose pulpar, e também a questão da condição socioeconômica.

Os dados relacionados à queixa principal demonstraram que a maior parte dos pacientes procurou o atendimento com a necessidade de fazer restauração, avaliação de rotina, presença da dor e fratura dentária. Esse resultado foi semelhante à pesquisa feita por Paula et al. ${ }^{18}$ realizada em um pronto atendimento de uma faculdade, tendo como principal relato quanto à queixa principal a dor, seguido da necessidade de procedimentos clínicos decorrentes de fratura, perda de restauração ou próteses provisórias e traumatismos dentários. Entre os procedimentos realizados, os mais frequentes foram restauradores. $O$ estudo de Tramini et al. ${ }^{19}$ mostrou que a dor aguda foi o motivo principal para a busca do atendimento odontológico.

Neste estudo, os dados referentes ao perfil clínico de todos os pacientes examinados através do prontuário, obteve-se a presença de dentes hígidos em maior quantidade, seguido da presença de Cárie, e Dentes ausentes. Para Schmidt et al. ${ }^{20}$ a cárie e a doença periodontal são predominantes na etiologia da perda dentária, e as duas são consideradas doenças crônicas, precisam além de um tratamento curativo, sendo restaurador ou endodôntico, também requerem sempre a avaliação rotineira para manter-se o estado de saúde bucal com condição dentária e periodontal permanentemente saudáveis.

No estudo de Silva-junior et al. ${ }^{21}$ quanto à frequência dos motivos autorrelatados para extração dentária, a dor e grande cavidade devido à cárie foram as mais relatadas com $37,5 \%$ e $9,3 \%$, respectivamente. Ramalho ${ }^{22}$ pesquisou no que se reporta às causas da origem dos problemas endodônticos, a etiologia que apareceu em destaque foi a cárie. Abdo et al. $^{23}$ fizeram uma pesquisa na qual relatava que 
a extração dentária é um procedimento feito com muita frequência e está comumente interligado com o baixo nível de instrução e/ou baixa classe socioeconômica.

Segundo Silveira et al. ${ }^{24}$ o baixo grau de escolaridade está associado às condições de saúde de maneira geral, em razão de influenciar nos cuidados preventivos e em relação aos conhecimentos sobre as doenças. Já pessoas com maior nível de escolaridade apresentam uma preocupação maior nos cuidados dos dentes. O estudo de Basting et al. $^{25}$ discorda desta pesquisa, dado que encontrou-se baixa incidência de dentes ausentes, pois em sua pesquisa obteve as médias dos componentes do CPOD, e observou que os componentes C (dentes cariados) e $\mathrm{O}$ (dentes restaurados) prevaleceram, uma vez que os resultados apresentaram-se praticamente nulos quando se analisou o componente $P$ isoladamente.

Quando se avalia o perfil clínico entre os gêneros, de forma separada, a presença de Cárie e Dentes Ausentes ainda superam as outras variáveis. Edman et al. ${ }^{26}$ afirmam, apesar de todos os esforços do Sistema Único de Saúde para a universalidade e a integralidade do atendimento, inclusive para melhoria da saúde bucal dos brasileiros, vemos ainda que a extração dentária devido a cárie ainda é uma condição muito prevalente, principalmente entre adultos e idosos.

Em análise comparativa ao perfil clínico masculino e feminino destacamos a presença da fratura dentária e de lesões cervicais não cariosas, o gênero masculino mesmo estando em menor quantidade no estudo, apresentou uma quantidade maior de números de dentes com fratura e lesões cervicais. O estudo de Carvalho et al. ${ }^{27}$ corrobora com os resultados dessa pesquisa, $64.6 \%$ dos pacientes acometidos por fratura dentária eram do gênero masculino e $35.4 \%$ do gênero feminino.

Caldas e Burgos $^{28}$ explicam que os homens são mais enérgicos e tendenciosos para atividades radicais, já que possuem uma vida social com trabalho e esporte mais perigosos, à medida que as mulheres são mais cuidadosas e maduras. Além do mais, o sexo masculino tem relatado uma possível associação entre níveis mais elevados de hormônios como a adrenalina, dopamina e hormônios relacionados ao estresse emocional, acarretando uma maior incidência em trauma. No que se refere à presença de lesões cervicais não cariosas, o estudo de Lima et al. ${ }^{29}$ divergiu desta pesquisa, pois as lesões foram mais comuns em mulheres. Por outro lado, na pesquisa de Oliveira et al. ${ }^{30}$ em relação ao gênero masculino e feminino, não houve diferença quanto à presença de Lesão Cervical não Cariosa.

No tocante a finalização do tratamento indicado, nesta pesquisa, a maior parte dos pacientes não concluíram 0 tratamento planejado, tanto homens como mulheres. Sugere-se que os motivos sejam pela ausência de controle com relação a organização dos prontuários, por o paciente precisar de outros tipos de tratamentos e o prontuário ficar em outras pastas, causando uma dinâmica difícil entre as clínicas de especialidades diferentes, ou também porque atendimentos realizados em clínicas-escola demoram mais tempo para serem concluídos, podendo acarretar em desistência do paciente, fazendo com que busque atendimento em clínicas particulares ou no serviço público.

O estudo de Ramalho ${ }^{22}$, embora tenha sido feito na mesma instituição, mas em clínica escola diferente, na de Endodontia da UFCG, apresentou resultado diferente deste estudo feito na Clínica de Dentística, pois a maior parte dos casos o procedimento foi finalizado com sucesso, enquanto o deste estudo a maioria não concluiu o tratamento.

Com isso, torna-se fundamental coletar dados que permitam traçar um planejamento de medidas preventivas e interceptativas ${ }^{31}$ diante do perfil de pacientes atendidos, tendo em vista que o estudante de odontologia precisa estar preparado para buscar compreender a condição de saúde bucal e planejar meios que gerem transformação social. Essas informações fornecem uma contribuição e mensuração sobre a relevância epidemiológica da condição de saúde bucal no serviço odontológico para que o aluno entenda a importância da prevenção de doenças e promoção de saúde, assim buscando meios indispensáveis ao progresso e à procura contínua de melhoria dos serviços.

CONCLUSÃO

Em face do que foi exposto nesta pesquisa, constata-se que a grande parte dos pacientes atendidos na clínica-escola de Dentística da Universidade Federal de Campina Grande é do gênero feminino, estando na faixa etária entre 31 e 45 anos, apresentando como queixas principais: fazer restauração; avaliação de rotina e sintomatologia dolorosa.

Quanto ao perfil clínico dos pacientes, as variáveis que mais se destacaram foram a presença de dentes hígidos, cariados e dentes ausentes. A perda do primeiro molar permanente demonstrou um resultado significativo em relação ao número de dentes ausentes. 
Constatou-se, ainda, que de acordo com o planejamento e os procedimentos executados nos pacientes, foi possível notar que a maioria dos pacientes não conclui o tratamento indicado, sugeriu-se que os motivos sejam pela ausência de controle com relação à organização dos prontuários, por o paciente precisar de outros tipos de tratamentos em outras clínicas de especialidades diferentes, ou também porque atendimentos realizados em clínicasescola demoram mais tempo para serem concluídos, podendo acarretar em desistência do paciente, buscando atendimento em outros serviços, seja público ou privado.

\section{REFERÊNCIAS}

1. Andriola FP, Toassi RFC, Paris MF, Baraldi CEE, Freddo AL. Perfil sociodemográfico, epidemiológico e comportamental dos pacientes atendidos no ambulatório de exodontia da FO-UFRGS e efetividade dos atendimentos realizados. Arq Odontol. 2015;51(2):104-15.

2. Domingos PAS, Rossato EM, Bellini A. Levantamento do perfil social, demográfico econômico de pacientes atendidos na clínica de Odontologia do Centro Universitário da Araraquara. Uniara. 2014;17(1):37-50.

3. Amorin NA, Silva TRC, Santos LM, Tenório $\mathrm{MDH}$, Reis JIL. Urgência em Odontopediatria: Perfil de Atendimento da Clínica Integrada Infantil da FOUFAL. Pesqui Bras Odontoped Clin Integr. 2007;7(3):223-27.

4. García-Pérez Á, Irigoyen-Camacho ME, Borges-Yáñez SA, Zepeda-Zepeda MA, Bolona-Gallardo I, Maupomé G. Impact of caries and dental fluorosis on oral healthrelated quality of life: a cross-sectional study in schoolchildren receiving water naturally fluoridated at above-optimal levels. Clin Oral Investig. 2017;21(9):2771-780.

5. Mota-Veloso I, Soares ME, Alencar BM, Marques LS, Ramos-Jorge MI, Ramos-Jorge J. Impact of untreated dental caries and its clinical consequences on the oral health-related quality of life of schoolchildren aged 8-10 years. Qual Life Res. 2016;25(1)193-99.

6. Marcenes W, Kassebaum NJ, Bernabe E, Flaxman A, Naghavi M, Lopez A et al. Global burden of oral conditions in 1990-2010: a systematic analysis. J Dent Res. 2013;92(7): 592-97.

7. Priyank H, Verma A, Gupta K, Chaudhary E, Khandelwal D, Nihalani S. In vitro comparative evaluation of various restorative materials used for restoring Class III cavities in deciduous anterior teeth: a clinical study. J Contemp Dent Contemp Dent Pract 2016;17(12):1022-26.

8. González J, Manrrique R. Proyecto AnacoU.C.V. estudio epidemiológico sobre la pérdida prematura del primer molar permanente en niños con edades comprendidas entre 6 y 10 años. Acta odontol. venez. 2001;39(2):42-6.

9. Antunes LS, Soraggi MBS, Antunes LAA, Corvino MPF. Avaliação e percepção das crianças econhecimento dos educadores frente à saúde bucal,dieta e higiene. Pesq Bras Odontoped Clín Integ. 2006;6(1):71-7.

10. Kanegane K, Penha SS, Borsatti MA, Rocha RG. Ansiedade ao tratamento odontológico em atendimento de urgência. Rev Saúde Publica. 2003;37:786-92.

11. Silva CHV, Araújo ACS, Fernandes RSM, Alves KA, Pelinca RN, Dias YC. Perfil do serviço de pronto atendimento odontológico da Universidade Federal de Pernambuco Odontol clín-cient. 2009;8(3):229-35.

12. Flumignan JDP, Sampaio Neto LF. Atendimento odontológico em unidades de emergência: caracterização da demanda. Rev Bras Odontol. 2014;71(2):124-29.

13. Xavier, CRG. Perfil epidemiológico dos pacientes atendidos nas clínicas da Disciplina de Cirurgia da Faculdade de Odontologia de Bauru da Universidade de São Paulo [dissertação]. Bauru: Curso de Odontologia, Faculdade de Odontologia de Bauru - USP; 2003.

14. Reis S, Santos L, Leles C. Clínica Integrada de Ensino Odontológico: Perfil dos usuários e necessidades odontológicas. ROBRAC. $2011 ; 20(52): 46-51$.

15. Ferreira Júnior $O$. Contribuição social do serviço de urgência odontológica da faculdade de Odontologia de Bauru - sua participação no convênio com sistema único de saúde [dissertação]. Bauru: Faculdade de Odontologia de Bauru, Universidade de São Paulo; 1997.

16. Gomes R, Nascimento EF, Araújo FC. Por que os homens buscam menos os serviços de saúde do que as mulheres? As explicações de homens com baixa escolaridade e homens com ensino superior. Cad Saúde Pública. 2007;23(3):565-74.

17. Silva AL, Saintrain MVL. Interferência do perfil epidemiológico do idoso na atenção odontológica. Rev Bras Epidemiol. 2006; 9(2):242-50.

18. Paula JS, Oliveira M, Soares MRSP; Chaves MGAM, Mialhe FL. Perfil epidemiológico dos pacientes atendidos no Pronto Atendimento da Faculdade de Odontologia da Universidade Federal de Juiz de Fora. Arq Odontol. 2012; 48(4):257-62.

19. Tramini P, Al Qadi Nassar B, Valcarcel J, Gibert $P$. Factors associated with the use of emergency dental care facilities in a French public hospital. Spec Care Dentist. 2010;30(2):66-71.

20. Schmidt MI, Duncan BB, Azevedo e Silva G, 
Menezes AM, Monteiro CA, Barreto SM, Chor D, Menezes PR. Chronic non-communicable diseases in Brazil: burden and current challenges. Lancet. 2011;377(9781):1949-61.

21. Silva-Junior MF, Sousa ACC, Batista MJ, Sousa MDLR. Condição de saúde bucal e motivos para extração dentária entre uma população de adultos (20-64 anos). Ciênc Saúde Colet.2017;22(8):2693-702.

22. Ramalho, JL. Perfil epidemiológico dos pacientes atendidos na clínica-escola de endodontia da universidade pública no Sertão da Paraíba [monografia]. Curso de Odontologia, Centro de Saúde e Tecnologia Rural, Universidade Federal de Campina Grande, Patos; 2017.

23. Abdo EM, Lima RPE, Rodrigues AS, Alves LCF, Gomes CO, Passos JB et al. Perfil do atendimento e dos pacientes usuários das clínicas de exodontias da Faculdade de Odontologia da Universidade Federal de Minas Gerais (UFMG). Arq Odontol. 2004;40(2): 183-95.

24. Silveira RG, Brum SC, Silva DC. Influência dos fatores sociais educacionais e econômicos na saúde bucal das crianças. RMAB. 2002; 52(1):34-9

25. Basting RT, Pereira AC, Maneghim MC. Avaliação da prevalência de cárie dentária em escolares do município de Piracicaba, SP, Brasil, após 25 anos de fluoretação das águas de abastecimento público. Rev Odontol Univ São Paulo. 1997;11(4):287-92.

26. Edman K, Ohrn K, Holmlund A, Nordström B, Hedin M, Hellberg D. Comparison of oral status in an adult population 35-75 year of age in the county of Dalarna, Sweden in 1983 and 2008. Swed Dent J. 2012;36(2):61-70.

27. Carvalho, RG; Soares SR, Silva EJNL, Mendonça TA, Antunes OHSFH, De-Deus G, Neves AA, Moreira EJL. Estudo epidemiológico das fraturas coronárias em pacientes atendidos em um projeto de trauma dental em um período de 6 anos. Rev Bras Odontol. 2013;70(1):4-7.

28. Caldas Jr AF, Burgos MEA. A retrospective study of traumatic dental injuries in a Brazilian dental trauma clinic. Dent. Traumatol. 2001;17: 250-55.

29. Lima LMD, Humerez Filho H, Lopes MDGK. Contribuição ao estudo da prevalência, do diagnóstico diferencial e de fatores etiológicos das lesões cervicais não-cariosas. RSBO. 2005;2(2):17-21.

30. Oliveira ACS, Damascena NP, Souza CS. Análise clínica de pacientes portadores de lesões cervicais cariosas e sua relação com hábitos. RSBO. 2010;7(2):182-92.

31. Almeida MDA, Sousa AL, Lemos ASC, Queiroz FS, Costa LED. Atendimento Odontopediátrico na Clínica-Escola de Odontologia da
Universidade Federal de Campina Grande (UFCG): perfil do paciente e necessidades assistidas. Arch Health Invest. 2019;8(9): 472-78.

\section{CONFLITO DE INTERESSES}

Os autores declaram não haver conflitos de interesse

\section{AUTOR PARA CORRESPONDÊNCIA}

\author{
José Henrique de Araújo Cruz \\ Rua Paulo Diogenes, 57 - Centro \\ 59990-000 Rafael Fernandes - RN, Brasil \\ Telefone: (83) 99625-0125. \\ E-mail: henrique_araujo1992@hotmail.com
}

Submetido em 08/04/2020 Aceito em 23/10/2020 